\title{
Neural correlates of late positivities associated with infrequent visual events and response errors
}

\section{Helenius, Paivi}

2010

Helenius , P , Laasonen , M , Hokkanen , L , Paetau , R \& Niemivirta , M 2010 , ' Neural correlates of late positivities associated with infrequent visual events and response errors ', Neurolmage , vol. 53 , no. 2 , pp. 619-628 . https://doi.org/10.1016/j.neuroimage.2010.06.059

http://hdl.handle.net/10138/252771

https://doi.org/10.1016/j.neuroimage.2010.06.059

publishedVersion

Downloaded from Helda, University of Helsinki institutional repository.

This is an electronic reprint of the original article.

This reprint may differ from the original in pagination and typographic detail.

Please cite the original version. 


\title{
Neural correlates of late positivities associated with infrequent visual events and response errors
}

\author{
Päivi Helenius ${ }^{\mathrm{a}, *}$, Marja Laasonen $^{\mathrm{b}, \mathrm{c}}$, Laura Hokkanen ${ }^{\mathrm{b}}$, Ritva Paetau ${ }^{\mathrm{d}}$, Markku Niemivirta ${ }^{\mathrm{e}}$ \\ a Brain Research Unit, MEG Core, Low Temperature Laboratory, Aalto University, Espoo, Finland \\ b Section of Psychology, Institute of Behavioural Sciences, University of Helsinki, Helsinki, Finland \\ c Department of Phoniatrics, Helsinki University Central Hospital, Helsinki, Finland \\ d Department of Pediatric Neurology, Helsinki University Central Hospital, Helsinki, Finland \\ e Section of Education, Institute of Behavioural Sciences, University of Helsinki, Helsinki, Finland
}

\section{A R T I C L E I N F O}

\section{Article history:}

Received 22 April 2010

Revised 17 June 2010

Accepted 23 June 2010

Available online 28 June 2010

\begin{abstract}
A B S T R A C T
The P3 response has been one of the most extensively studied event-related potential (ERP) components. Still, the exact functional role and cortical basis of P3 has remained unsettled. To explore the cortical processes underlying the generation of late positivities, we recorded the activation evoked by frequent Go and infrequent NoGo stimuli and correct versus erroneous responses using combined magnetoencephalography (MEG) and ERP measurements during a visual Go/NoGo task. The stimulus-locked signals in the ERP channels revealed an enhanced negative N2 and a prominent late positive component (LPC) after the complex NoGo stimuli associated with successfully withheld responses. The response-locked ERP signals revealed error-related negativity (ERN) and positivity (Pe) after erroneous responses. The positive LPC and Pe components were coupled with functionally and temporally comparable MEG signals. This MEG activation detected during the positive components was localized bilaterally in the posterior temporal cortex. In the response-locked averages, the temporal activity was enhanced around $200 \mathrm{~ms}$ after a commission of an error. In the stimuluslocked averages, the activation was also enhanced after infrequent NoGo stimuli around $500 \mathrm{~ms}$ after stimulus onset and delayed about $80 \mathrm{~ms}$ for the initially miscategorized NoGo stimuli accompanied by erroneous response. The results suggest that the cortical correlates of Pe are not specifically related to commission of an error, but both the LPC and Pe components, and bilateral temporal cortices, are more generally involved in stimulus-driven attentional processing evoked by unexpected stimuli. The negative ERP components evoked by NoGo stimuli (N2) and erroneous responses (ERN) were found to be associated with partly non-overlapping neural sources.
\end{abstract}

(c) 2010 Elsevier Inc. All rights reserved.

\section{Introduction}

When the task requires the subject to respond to a certain category of stimuli (Go) and to withhold a response to another category of stimuli (NoGo), event-related potential (ERP) studies commonly report differences between the trials during two distinct deflections: The N2 deflection peaking around $300 \mathrm{~ms}$ is more negative to NoGo than to Go stimuli, and P3, a broad positive component with a typical peak latency between 300 and $400 \mathrm{~ms}$, is enhanced in amplitude or latency to NoGo stimuli (Pfefferbaum et al., 1985; Kok, 1986; Nieuwenhuis et al., 2003; Amodio et al., 2008). When the averaging is done time-locked to correct and erroneous responses, an error-related negativity (ERN) peaking 60 $80 \mathrm{~ms}$ after incorrect response and error positivity (Pe) maximal between 200 and $400 \mathrm{~ms}$ are commonly reported (Gehring et al., 1990, 1993; Falkenstein et al., 1991; Overbeek et al., 2005). The

\footnotetext{
* Corresponding author. Brain Research Unit, MEG Core, Low Temperature Laboratory, Aalto University, P.O. Box 15100, 00076 Aalto, Finland. Fax: + 358947022969.

E-mail address: paivi@neuro.hut.fi (P. Helenius).
}

cognitive and neural processes associated with these negative and positive components in Go/NoGo tasks are not yet thoroughly understood.

The ERN component that follows error commission is viewed as reflecting activity of a response monitoring system (Falkenstein et al., 1991; Gehring et al., 1993; Botvinick et al., 2004) possibly sensitive to the motivational significance of an error (Hajcak et al., 2005). Anterior cingulate cortex (ACC) is a relatively well-established generator of the ERN (Dehaene et al., 1994; Carter et al., 1998). The neural basis of the negative component associated with successfully inhibited responses in NoGo trials is less clear. According to one view, the N2 is also produced in ACC as a response to the presence of a conflict (Nieuwenhuis et al., 2003; Yeung et al., 2004; Amodio et al., 2008), while according to a quite different view, N2 is related to the detection of novelty of perceptual or mental template with unknown cortical source (Nieuwenhuis et al., 2004; Folstein and Van Petten, 2008).

In a similar vein, the late positivities have been argued to reflect either stimulus-driven or error-centered processes. The error-centered hypothesis of Pe relates this component to emotional appraisal, 
conscious recognition or performance adjustment following an error (Falkenstein, 2004) with possible neural source in the posterior cingulate regions (Vocat et al., 2008). However, some studies based on the comparable morphology, topology and age-related changes in P3 and Pe responses suggest that the late positivities have the same neural source (for a review, see Overbeek et al., 2005). P3 is related to stimulusdriven factors, especially to the probability of occurrence (DuncanJohnson and Donchin, 1977) and task or stimulus complexity (McCarthy and Donchin, 1981); the less probable the stimulus category the larger the P3, and the harder the task the later the P3. Thus it has been suggested that P3 is related to updating or revision of mental representation in working memory induced by incoming stimulus (Donchin, 1981; Donchin and Coles, 1988; Polich, 2007) or the decision about how to respond to the stimulus (Nieuwenhuis et al., 2005; Verleger et al., 2005).

The neural source of P3/Pe deflection is unknown, although based on the wide scalp distribution, several sources are assumed to contribute to the measured signal. Accordingly, generators of the visual P3 potential have been reported in deep mesial temporal structures (Goto et al., 1996; Mecklinger et al., 1998) but also in inferior frontal, inferior temporal and parietal areas (Yamazaki et al., 2000; Moores et al., 2003; Bledowski et al., 2004). Intracranial ERP studies using depth electrodes implanted in the brain in epileptic patients have detected largest P3 generators in the hippocampus but well-established local generators are also found in the superior temporal sulcus and ventro-lateral prefrontal cortex (Baudena et al., 1995; Halgren et al., 1995a,b, 1998). Patient studies have convincingly shown that lesions of the temporo-parietal junction lead to reduced auditory and visual P3 component (Knight et al., 1989; Verleger et al., 1994) while after bilateral hippocampal lesions the P3 component is not as clearly affected (Polich and Squire, 1993). In fMRI studies, low probability visual stimuli activate areas in the lateral posterior occipital cortex and parietal, temporal and prefrontal cortex (McCarthy et al., 1997; Marois et al., 2000), although temporo-parietal junction seems to have a special role, for example, in multimodal detection of changes in the sensory environment (Downar et al., 2000).

Magnetoencephalography (MEG) is based on detecting weak magnetic fields associated with neural activation using sensitive sensors and can readily follow both the spatial and temporal patterns of cortical activation (Hämäläinen et al., 1993; Salmelin and Baillet, 2009). To our knowledge, only two previous MEG studies have attempted to reveal cortical sources of error-related activity with partly inconsistent results. In the Miltner et al. (2003) study, 31channel magnetometer was used to record activation during auditory Go/NoGo task. The task was replicated in each participant 2-6 times placing the channels in different locations around the head. In the six individuals measured, a localization based on difference waveform for incorrect and correct responses suggested a neural source for the ERN in ACC or closely adjacent areas. Using a whole-head MEG system, Stemmer et al. (2004) detected a more distributed network of brain regions activated during error trials, the main source of activation arising from sensorimotor area. The neural sources of N2 or late positivities were not investigated in either of these two MEG studies. Previous visual MEG experiments have localized P3/P300 activation in the vicinity of thalamus (Horiguchi et al., 2003), in the hippocampal formation and in parahippocampal, temporal and occipital extrastriate cortex (Basile et al., 1997) with no mentioning of the errorrelated components ERN or Pe.

In the current experiment we used MEG to further investigate the cognitive and physiological processes associated with visual Go/NoGo task. Concurrent ERP recordings were performed in order to identify critical time windows that reveal stimulus- or response-related modulations. Largest P3 components after infrequent stimuli have been detected using frequent Go and infrequent NoGo stimulus presentation (Eimer, 1993; Nieuwenhuis et al., 2003). We adopted this approach to maximize the chances of identifying the neural structures involved in P3 generation with MEG. We gathered both stimulus- and response-locked averages to gain full representation of shared or distinct neural processes related to P3 and Pe. Our design also allows to measure and compare error-related Pe components as well as the P3 components elicited by infrequent stimuli that are correctly or incorrectly reacted to. Deepened knowledge about the neural and cognitive basis of late positivities could further clarify the clinical and theoretical implications of some P3/Pe-related findings.

\section{Materials and methods}

\section{Participants}

We recorded data from 13 adults $19-48$ years of age (mean 29 years, 7 females). All participants were right-handed according to their own report. Informed consent was obtained from all subjects, in agreement with the prior approval of the Ethics Committee of the Hospital District of Helsinki and Uusimaa.

\section{Stimuli and experimental procedures}

Our stimuli were arrays composed of 5 visual items (apples and animals) (Fig. 1). The relative position of items varied between successive stimuli presented once every $2.2 \mathrm{~s}$. Display duration of each array was $150 \mathrm{~ms}$. The stimuli were presented on a rear projection screen placed in front of the subject sitting in the magnetically shielded room (Imedco AG, Hägendorf, Switzerland). All images were presented at the same central viewing position occupying a visual angle of about $6^{\circ} \times 1^{\circ}$ on a light grey background. The screen background color remained the same between the stimuli. The participants were instructed to make a rapid manual response to a target stimulus (wolf is facing a pig; probability 83\%) and avoid responding to a nontarget stimulus (wolf is not facing a pig; probability $17 \%$ ). The nonmagnetic response button rested in the participant's right hand. Trigger signals, coinciding with thumb flexions, were detected optically using an optoswitch and two plastic optic fibers connected to the response button. The game-like setup was developed to enhance the direct use of our stimuli in clinical settings and in studies of young children.

\section{Data acquisition}

During two approximately 12-minute sessions, 3 ERP channels and a whole-head MEG system were used to record: i) stimulus-locked signals and ii) response-locked signals. The stimulus-locked signals were averaged for target stimuli (Go correct trials) and separately for non-target stimuli that were correctly not responded to (NoGo correct trials) and to stimuli that were followed by an erroneous response (NoGo error trials). In both Go correct trials and NoGo error trials only those responses that followed the stimuli within $700 \mathrm{~ms}$ were included. Response-locked signals were averaged time-locked to those responses that followed the target stimuli within $700 \mathrm{~ms}$ (Go correct trials) and to erroneous responses following the non-target stimuli (NoGo error trials) within $700 \mathrm{~ms}$.

ERP signals were measured from $\mathrm{Fz}, \mathrm{Cz}$ and $\mathrm{Pz}$ according to the International 10-20 system (reference electrode $\mathrm{AFz}$, ground neck). Neuromagnetic brain responses were recorded using a 306-channel Elekta Neuromag ${ }^{\mathrm{TM}}$ neuromagnetometer (Elekta Oy, Helsinki, Finland) that measures magnetic field strength in 102 locations over the scalp (two planar gradiometers and one magnetometer at each location). Prior to measurements, four head-position indicator coils were attached to the subject's scalp, and the locations of these coils were determined in relation to three anatomical landmarks (preauricular points and nasion) with a 3-D digitizer. At the beginning of 


\section{GO STIMULI (83\%; wolf facing a pig) NOGO STIMULI (17\%; wolf not facing a pig)}

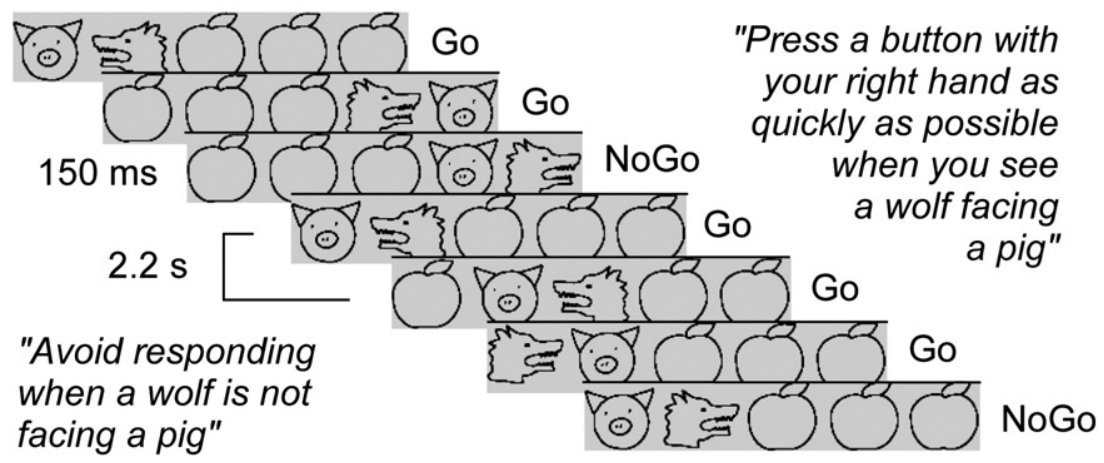

Fig. 1. The visual stimuli used in the study.

the experimental session, an electric current was fed to the coils and their locations with respect to the MEG helmet were measured.

The MEG and EEG signals were band-pass filtered at $0.1-200 \mathrm{~Hz}$ and sampled at $600 \mathrm{~Hz}$. Stimulus-locked responses were averaged from $200 \mathrm{~ms}$ before to $800 \mathrm{~ms}$ after the stimulus onset (Go correct trials, NoGo correct trials and NoGo error trials) and response-locked signals -500 before to $500 \mathrm{~ms}$ after the button press (Go correct trials, NoGo error trials). Averaged responses were low-pass filtered at $40 \mathrm{~Hz}$. Vertical and horizontal eye movements were continuously recorded and epochs contaminated by ocular signals were rejected $(\mathrm{EOG}>300 \mu \mathrm{V})$. The number of artifact-free trials was on average 472 for stimulus-locked Go correct trials, 68 for stimulus-locked NoGo correct trials, 28 for stimulus-locked NoGo error trials, 432 for response-locked Go correct trials and 27 for response-locked NoGo error trials.

\section{MEG data analysis}

We localized the active source areas using Minimum Current Estimates (MCE; Uutela et al., 1999) and Equivalent Current Dipole analysis (ECD analysis; Hämäläinen et al., 1993). For previous studies using combined MCE and ECD modeling, see Stenbacka et al. (2002) and Vartiainen et al. (2009). MCE accounts for the measured signals by a distribution of electric current that has the minimum total amplitude. MCEs were first calculated from the data of each individual participant for each condition and, thereafter, the MCEs for each condition were averaged across participants. In the Elekta Neuromag MCE program (Uutela et al., 1999), a region of interest is selected from the color display by selecting the approximate nodes of interest. The program will then calculate the ROI center and extent. We manually further adjusted the ROI extents calculated from group-averaged data to better account for individual data. The ROIs were calculated from those trials that showed the strongest activity within a potential brain volume around the peaks of the N2, ERN and positive components (majority of the ROIs identified during the N2 and positive components were determined from the stimulus-locked NoGo correct trials and during the ERN from the response-locked NoGo error trials). ECD analysis reduces the signals detected by the MEG sensors into the time behavior of distinct cortical areas. Each ECD represents the center of an active cortical patch and the strength and direction of electric current in that area. The analysis was done with the Elekta Neuromag software package, and proceeded as described earlier (e.g., Salmelin et al., 1996; Wydell et al., 2003; Parviainen et al., 2006; Vartiainen et al., 2009). Each ECD was determined at a time point where the dipolar field was most salient and had least interference from other active areas. Individually in each subject, for each distinct dipolar field pattern, a subset of planar gradiometer sensors was selected that spatially covered the field pattern, and the location of the neural population generating that response was determined. Thereafter, the locations and orientations of the ECDs ( 8 sources per participant in stimulus-locked trials and 6 dipoles in response-locked trials) were fixed while their amplitudes were allowed to vary to achieve maximal explanation of the recorded whole-head 306channel data in each condition. For an example of MEG data recorded from one subject and an associated multidipole model of 8 sources, see Supplementary data. Structural MR images were not available for the great majority of subjects. Therefore, a default model of the brain was used in the MCE analysis and the ECD sources of each individual are displayed on an average brain.

\section{Results}

Participants responded significantly slower to target stimuli (mean $419 \mathrm{~ms} \pm$ SD $37 \mathrm{~ms}$ ) than to non-target stimuli, i.e., when they made an error by responding to NoGo trial ( $361 \mathrm{~ms} \pm 29 \mathrm{~ms}$; paired $t$-test, $p<0.001$ ). Compared to a previous ERP study using relative simple stimuli with comparable Go/NoGo probability (Nieuwenhuis et al., 2003), the reaction times in the current study with relatively complex visual stimuli were overall about $100 \mathrm{~ms}$ slower. Performance accuracy on target trials was nearly perfect; the mean percentage of very slow responses in Go correct trials (>700 ms) was on average $2 \%$. Error rates in NoGo trials were on average $29 \%$ but varied considerably between participants (from 6 to $70 \%$ ).

\section{Analysis of the stimulus-locked ERP and MEG signals}

\section{ERP results-stimulus-locked signals}

The stimulus-locked ERP waveforms associated with target and non-target stimuli are shown in Fig. 2. The correct NoGo trials elicited a more negative going deflection compared to Go correct trials $390 \mathrm{~ms}$ after the stimulus presentation in all three channels. In previous ERP studies with simple stimuli and quicker response latencies, this N2 activation is detected around $300 \mathrm{~ms}$ (Nieuwenhuis et al., 2003). The NoGo error trials also elicited a negative deflection peaking around 400-420 ms, slightly after the N2 response. In each participant, the N2 was identified as the peak negative deflection occurring 330-430 ms after the onset of the stimuli in NoGo correct trials. As in one subject none of the three channels showed a distinct peak during the 330$430 \mathrm{~ms}$ time window, the time point of the maximal difference between NoGo correct and Go correct trials was used instead. The N2 response peaked on average within $7 \mathrm{~ms}$ in the three ERP channels (mean latency across channels $387 \mathrm{~ms} \pm 24 \mathrm{~ms}$ ). During the individually determined $\mathrm{N} 2$ peaks the responses were more negative in all three channels for NoGo correct trials $(0.3 \pm 2.7 \mu \mathrm{V}$ in Fz, $1.7 \pm 4.5 \mu \mathrm{V}$ 


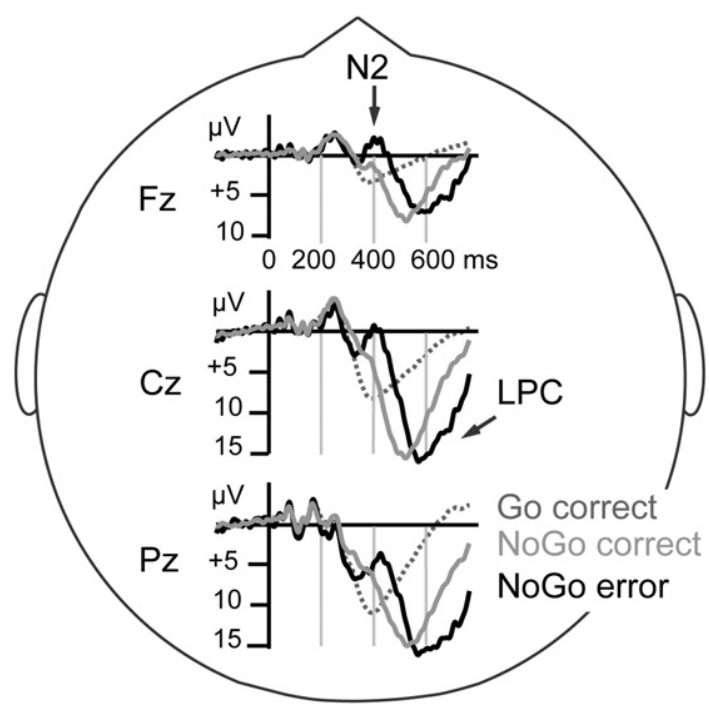

Fig. 2. ERP responses in electrodes $\mathrm{Fz}, \mathrm{Cz}$ and $\mathrm{Pz}$. The responses were averaged with respect to stimulus presentation across all subjects for Go correct trials (frequent target stimuli followed by correct response), NoGo correct trials (non-target stimuli associated with successfully withheld response) and NoGo error trials (non-target stimuli followed by erroneous response). N2 and LPC deflections are indicated with arrows.

in $\mathrm{Cz}$ and $4.9 \pm 4.6$ in $\mathrm{Pz})$ than to Go correct trials $(3.2 \pm 2.9 \mu \mathrm{V}$ in $\mathrm{Fz}$, $7.8 \pm 4.0 \mu \mathrm{V}$ in $\mathrm{Cz}$ and $10.4 \pm 4.8 \mu \mathrm{V}$ in Pz; paired $t$-test $p<0.001$ in all three channels). In addition, we compared the mean amplitudes of the NoGo and Go correct trials around the N2 peak (355-405 ms) after high-pass filtering the waveforms above $2 \mathrm{~Hz}$. Even when the effects of slow positivities seen around the time of the $\mathrm{N} 2$ response were removed (for a similar approach, see Jodo and Kayama, 1992), the difference between categories was statistically significant $(-1.8 \pm$ $2.3 \mu \mathrm{V}$ for NoGo correct trials and $3.2 \pm 1.1 \mu \mathrm{V}$ for Go correct trials in Cz; paired $t$-test $p<0.001$ ).
In a later time window, the NoGo correct trials elicited a broad positive component maximal in the averaged waveforms around $530 \mathrm{~ms}$ after the stimulus onset. In each participant this late positive component (LPC) was identified as the peak positive deflection occurring after $510 \mathrm{~ms}$ from the onset of the stimuli in the NoGo correct trials. During the LPC peak the responses were more positive in all three channels for the NoGo correct trials $(8.9 \pm 3.4 \mu \mathrm{V}$ in $\mathrm{Fz}$, $16.9 \pm 6.2 \mu \mathrm{V}$ in $\mathrm{Cz}$ and $16.5 \pm 5.6 \mathrm{in} \mathrm{Pz}$ ) than Go correct trials (1.5 \pm $2.9 \mu \mathrm{V}$ in $\mathrm{Fz}, 5.8 \pm 4.4 \mu \mathrm{V}$ in $\mathrm{Cz}$ and $5.1 \pm 3.9$ in $\mathrm{Pz}$; paired $t$-test $p<0.001$ in all three channels). A positive deflection was also evident after the NoGo error trials peaking approximately $50 \mathrm{~ms}$ later than the LPC for the NoGo correct trials.

\section{MCE analysis of MEG data-stimulus-locked signals}

We calculated the minimum current estimates around the N2 peak (355-405 ms) for the Go correct and NoGo correct trials (Fig. 3). Activation elicited by NoGo correct and Go correct trials was compared in four left hemisphere, four right hemisphere and one midline anterior regions of interest (ROI). Only one ROI centered in the right occipito-temporal border was more strongly activated during the N2 peak to NoGo correct than to Go correct trials (5.8 \pm $3.4 \mathrm{nAm}$ vs. $4.2 \pm 2.7 \mathrm{nAm}$, respectively; paired $t$-test $p<0.05)$. Stronger activation in the left primary somatosensory cortex was detected to Go correct trials than to NoGo correct trials already at this time window due to the right hand motor responses (button presses) associated with correct task performance $(6.8 \pm 4.7 \mathrm{nAm}$ vs. $4.9 \pm 3.1$ nAm, respectively; paired $t$-test $p<0.04)$.

MCE analysis around the peak of the LPC (475-575 ms) revealed enhanced activation in the bilateral posterior temporal cortex for NoGo correct $(5.5 \pm 5.8 \mathrm{nAm}$ in the left and $6.7 \pm 2.2 \mathrm{nAm}$ in the right hemisphere) compared to Go correct trials ( $3.3 \pm 3.7 \mathrm{nAm}$ in the left and $3.4 \pm 1.3 \mathrm{nAm}$ in the right hemisphere, paired $t$-test between Go correct and NoGo correct trials $p<0.01$ in the left hemisphere and $p<0.001$ in the right hemisphere) (Fig. 4). Enhanced activation was also detected for the NoGo error trials in these same ROIs $(5.2 \pm 3.8 \mathrm{nAm}$ in the left and $6.2 \pm 2.7 \mathrm{nAm}$ in the right hemisphere, paired $t$-tests $p<0.01$ between Go correct and NoGo error trials in both hemispheres).
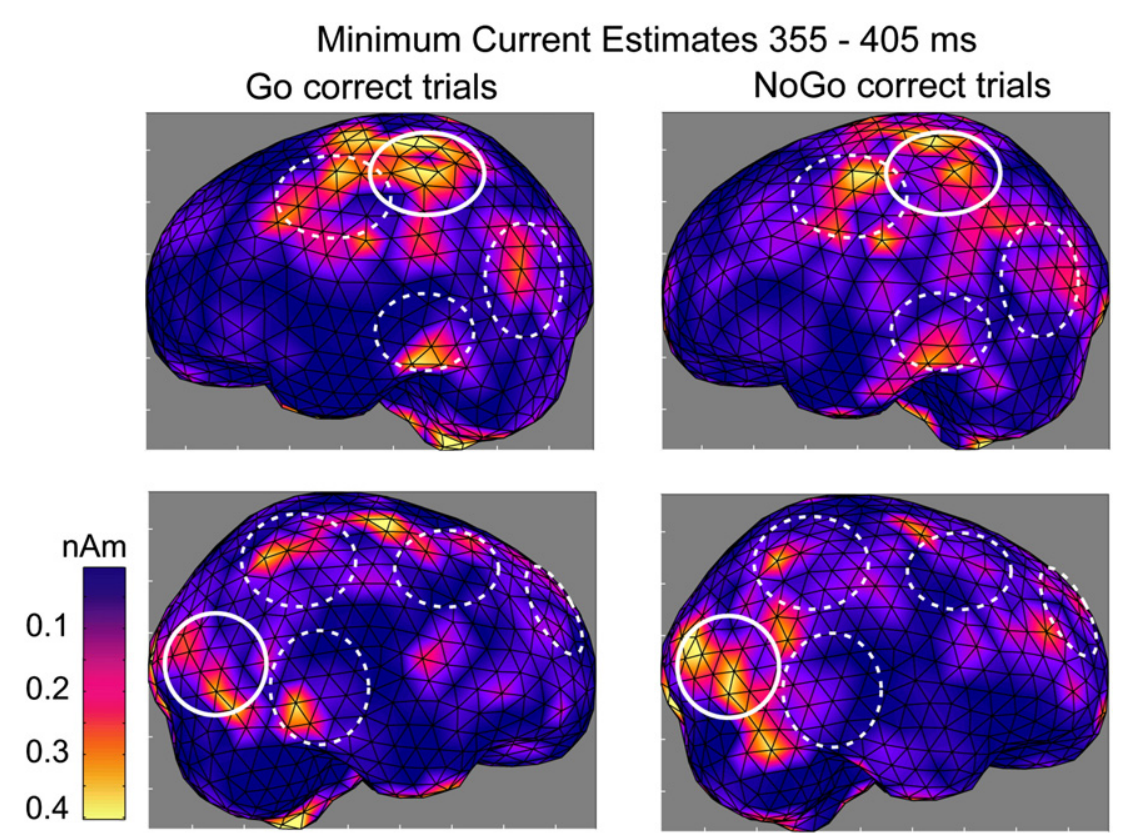

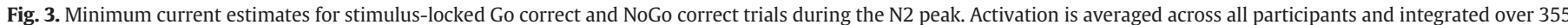

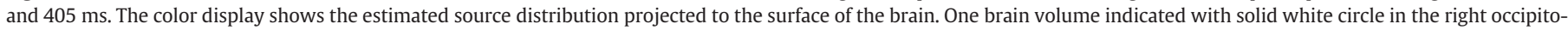

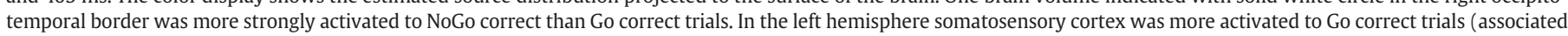
with the button press) than to NoGo correct trials. The dashed circles indicate statistically non-significant differences between the two trials. 


\section{Minimum Current Estimates 475 - 575 ms}

Go correct trials
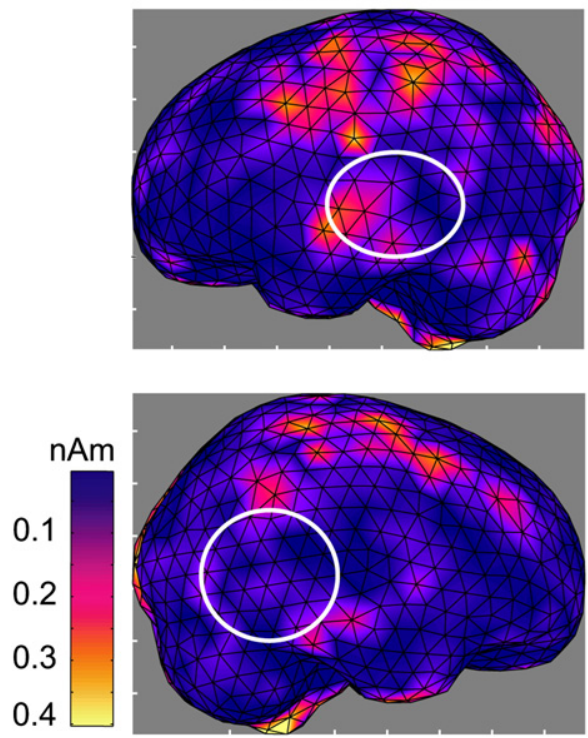

NoGo correct trials
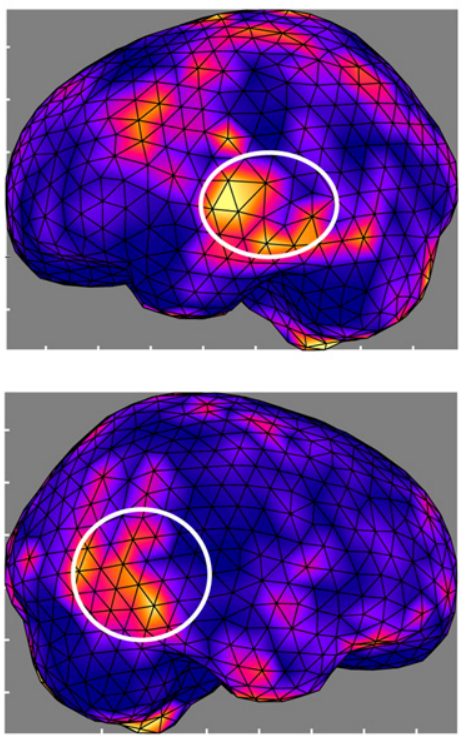

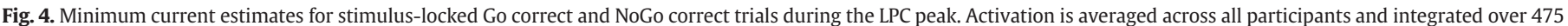
and $575 \mathrm{~ms}$. The brain volumes in the bilateral posterior temporal cortices showed stronger activation to NoGo correct than Go correct trials (solid white circles).

\section{ECD analysis of MEG data-stimulus-locked signals}

Fig. 5 shows the distribution and time behavior of those equivalent current dipoles across all participants that centered in the left and right posterior temporal areas spatially overlapping with the activation detected during LPC in the MCE analysis. In 10/13 participants in the left and in $11 / 13$ participants it the right hemisphere the mean signal strength of these ECDs during the LPC activation (475-575 ms) was stronger to NoGo correct than Go correct trials (i.e., the difference between categories was at least 2.0 times the baseline period $200 \mathrm{~ms}$ prior the stimulus). In these same ECD the mean activation to NoGo correct trials also exceeded 2.0 times the baseline activation and was above $6 \mathrm{nAm}$. Similar significant ECD activation that was stronger to NoGo than Go correct trials was not detected as consistently across participants in other cortical areas; the difference between categories reached statistical significance around the LPC response in few individuals in ECDs that were scattered in the mid occipital area (4 participants), in the right lateral occipital area (3 participants) and in left lateral occipital area (3 participants) and in somatosensory area (one participant). Around the peak of the LPC (475-575 ms) the mean
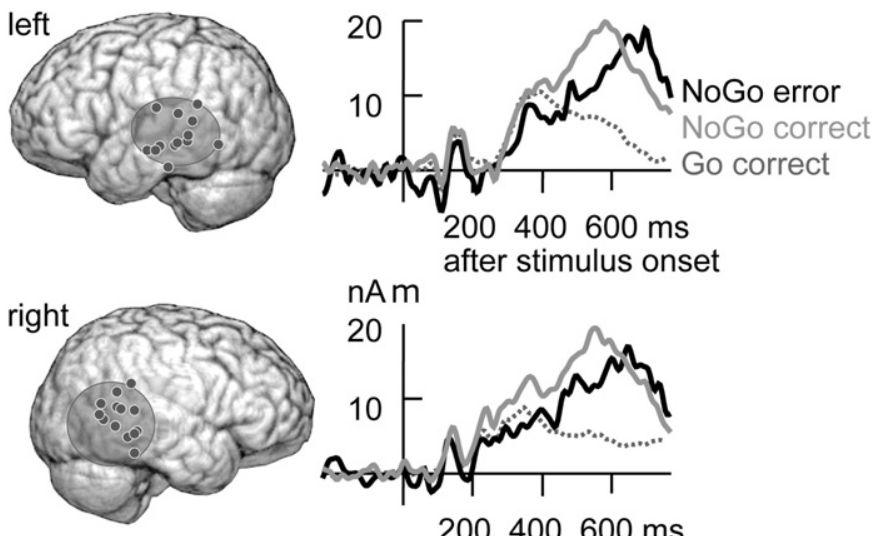

$\mathrm{nAm}$

after stimulus onset

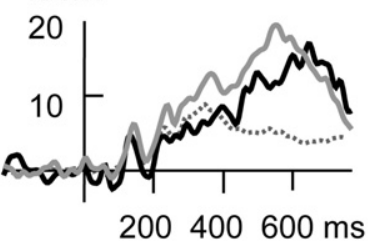

Fig. 5. Left : The distribution of equivalent current dipoles across all subjects in the left and right temporal cortex shown on an atlas brain. Right: The mean time courses of activation averaged across all subjects for stimulus-locked Go correct, NoGo correct and NoGo error trials in the left and right temporal areas. activation across all temporal dipoles was stronger to NoGo correct trials $(18.0 \pm 14.3 \mathrm{nAm}$ in the left and $18.2 \pm 12.0 \mathrm{nAm}$ in the right hemisphere) than to Go correct trials $(7.4 \pm 7.5 \mathrm{nAm}$ in the left and $5.5 \pm 4.7 \mathrm{nAm}$ in the right hemisphere, paired $t$-test $p<0.002$ in the left and $p<0.001$ in the right hemisphere).

The mean peak latency of activation in the temporal areas calculated across ECDs displaying stronger activation to NoGo correct than Go correct trials was $553 \mathrm{~ms}( \pm 52 \mathrm{~ms})$ in the left hemisphere and $533 \mathrm{~ms}( \pm 44 \mathrm{~ms})$ in the right hemisphere (non-significant difference between the hemispheres). The activation elicited by NoGo error trials in the left and right temporal areas was delayed by about $80 \mathrm{~ms}$ compared to the activation elicited by NoGo correct trials (paired $t$-test $p<0.01$ in both hemispheres).

Particularly in the right hemisphere it appeared that the activation had also an earlier peak around the time when the N2 deflection peaked at ERP data $390 \mathrm{~ms}$ after stimulus onset. In three subjects also a separate right hemisphere occipito-temporal dipolar source explained the activation during the $\mathrm{N} 2$ time window. When the activation was compared between NoGo correct trials and Go correct trials in the right hemisphere in these 3 subjects with occipito-temporal and in the remaining subjects with posterior temporal source, the activation for NoGo correct trials $(15.3 \pm 8.1 \mathrm{nAm})$ was stronger than for the Go correct trials between $(355-405 \mathrm{~ms})(9.5 \pm 5.1 \mathrm{nAm}$, paired $t$-test $p<0.01$ ).

In summary, the stimulus-locked signals in ERP channels revealed well-known negative N2 and positive LPC deflections associated with the NoGo correct trials. During the N2 deflection, 355-405 ms after stimulus onset, right occipito-temporal border was activated more strongly to NoGo correct trials than to Go correct trials. During the LPC response, 475-575 ms after stimulus onset, bilateral temporal cortices showed a stronger activation to NoGo correct than Go correct trials. This activation was also delayed for the NoGo error trials, i.e. initially miscategorized non-targets followed by an erroneous response.

Analysis of the response-locked ERP and MEG signals

ERP results-response-locked signals

The response-locked ERP waveforms associated with the Go correct trials and NoGo error trials are shown in Fig. 6. A negative deflection peaking $60 \mathrm{~ms}$ after the erroneous response was evident in all three 


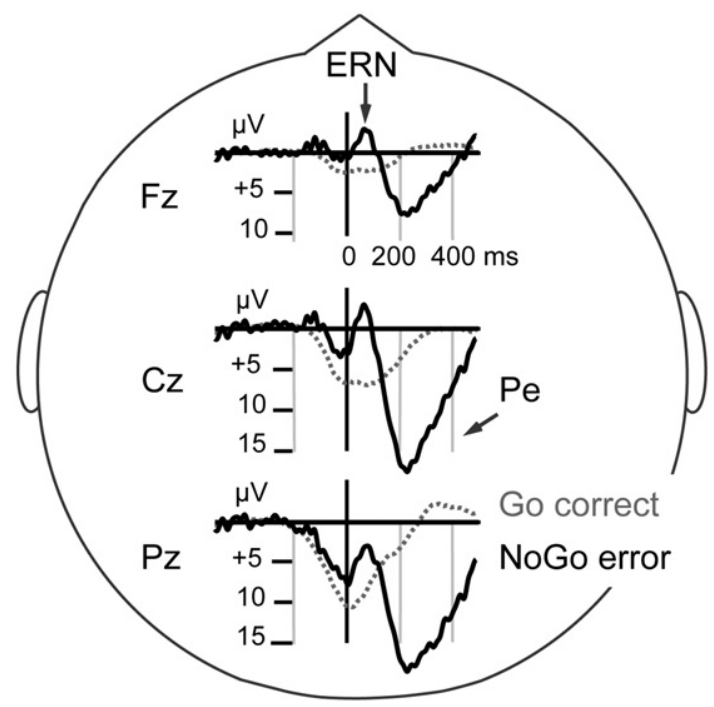

Fig. 6. ERP responses in electrodes $\mathrm{Fz}, \mathrm{Cz}$ and $\mathrm{Pz}$. The responses were averaged with respect to button press across all subjects for Go correct trials (correctly responded frequent targets) and NoGo error trials (erroneously responded infrequent nontargets). ERN and Pe deflections are indicated with arrows.

channels. In each participant this ERN response was identified as the peak negative deflection occurring $30-130 \mathrm{~ms}$ after the erroneous button press. During the ERN peak the responses were more negative in all three channels for NoGo error trials $(-4.8 \pm 5.9 \mu \mathrm{V}$ in $\mathrm{Fz},-4.7$ $\pm 8.9 \mu \mathrm{V}$ in $\mathrm{Cz}$ and $0.7 \pm 8.2 \mathrm{in} \mathrm{Pz})$ than to Go correct trials $(2.2 \pm 2.3 \mu \mathrm{V}$ in $\mathrm{Fz}, 6.9 \pm 4.5 \mu \mathrm{V}$ in $\mathrm{Cz}$ and $8.5 \pm 5.2$ in Pz, paired $t$-test $p<0.001$ in all three channels).

In a later time window the NoGo error trials elicited a broad positive component maximal in the averaged waveforms around $230 \mathrm{~ms}$ after erroneous button press. In each participant this Pe component was identified as the peak positive deflection occurring $180-280$ ms from the onset of the button press. During the Pe peak the responses were more positive in all three channels for NoGo error trials $(8.8 \pm 4.6 \mu \mathrm{V}$ in Fz, $18.9 \pm 8.9 \mu \mathrm{V}$ in $\mathrm{Cz}$ and $20.0 \pm 10.2 \mathrm{in} \mathrm{Pz}$ ) than Go correct trials $(-0.1 \pm 1.9 \mu \mathrm{V}$ in $\mathrm{Fz}, 2.7 \pm 2.5 \mu \mathrm{V}$ in $\mathrm{Cz}$ and $1.9 \pm 2.4$ in Pz, paired $t$-test $p<0.001$ in all three channels).

\section{MCE analysis of MEG data-response-locked signals}

In the evaluation of the error-related MEG signals, the fairly low overall frequency of erroneous responses should be kept in mind (mean 27 artifact-free NoGo error trials, 9 out of 13 participants had below 28 errors), as consequently, the error-related source estimates are relatively noisy. We calculated the minimum current estimates around the ERN peak (30-90 ms) and compared the activation elicited by NoGo error trials and Go correct trials in two left hemisphere, three right hemisphere and midline anterior ROIs (Fig. 7). After erroneous responses stronger activation was detected in the right occipito-temporal border $(5.2 \pm 2.7 \mathrm{nAm}$ vs. $3.1 \pm 2.1$ $\mathrm{nAm}$, paired $t$-test $p<0.01)$, right frontal area $(3.7 \pm 3.1 \mathrm{nAm}$ vs. $1.9 \pm$ $2.0 \mathrm{nAm}, p<0.01)$ and anterior midline area $(5.7 \pm 4.5 \mathrm{nAm}$ vs. $2.7 \pm$ $3.5 \mathrm{nAm}, p<0.003$ ) compared to correct responses. The motor responses (right hand button presses) associated with errors elicited stronger activation in the left primary somatosensory cortex than those motor responses that were associated with correct task performance (NoGo error trials $8.7 \pm 5.4 \mathrm{nAm}$ vs. Go correct trials $6.9 \pm 4.7 \mathrm{nAm}$, paired $t$-test $p<0.05)$. Further, the mean signal strength in each of these ROIs was highly statistically significant for the NoGo error trials during the ERN peak (at least 3.3 times stronger signal 30-90 $\mathrm{ms}$ after the response than during the baseline period at 500-300 ms prior the response). Thus, the observed differences between erroneous and correct responses in the 3 ROIs are not reflecting the overall higher noise level of the NoGo error trials.

MCE analysis around the peak of the Pe (180-280 ms) revealed enhanced activation in the bilateral posterior temporal cortex for NoGo error trials $(5.7 \pm 5.1 \mathrm{nAm}$ in the left and $5.8 \pm 2.6 \mathrm{nAm}$ in the right hemisphere) compared to Go correct trials (3.4 $\pm 3.1 \mathrm{nAm}$ in the left and $3.1 \pm 1.1 \mathrm{nAm}$ in the right hemisphere, paired $t$-test between NoGo error trials and Go correct trials $p<0.03$ in the left hemisphere and $p<0.002$ in the right hemisphere) (Fig. 8). In addition, the left frontal cortex around the left premotor area was more strongly activated after erroneous than correct responses $(3.8 \pm 3.3 \mathrm{nAm}$ vs. $1.5 \pm 1.2 \mathrm{nAm}$, paired $t$-test $p<0.05)$. The mean signal strength in the two left hemisphere ROIs was highly statistically significant for the NoGo error trials during the Pe peak (at least 3.3 times stronger signal 180-280 ms after the response than during the baseline period at 500-300 ms prior the response). In the right hemisphere, the signal strength in NoGo error trials reached only the 0.01 significance level.

\section{ECD analysis of MEG data-response-locked signals}

The dipole analysis performed on the individual data was also challenged by the fairly low overall frequency of erroneous responses. Particularly during the ERN activation no field patterns suitable for dipole modeling were detected in the anterior midline or frontal regions except for one subject. In the temporal area however, 11 subjects showed clear activation around the same time the Pe response peaked in the ERP data in both the left and in the right hemisphere temporal areas. This activation was explained using the same set of dipoles that were localized in the stimulus-locked data using the responses following the NoGo correct trials. Fig. 9 shows the mean time behavior of the left and right posterior temporal areas. In individual subjects this activation peaked at very variable latencies ranging from 180 to $380 \mathrm{~ms}$. On average the maximal activation in the temporal areas was detected at $294 \mathrm{~ms}( \pm 68 \mathrm{~ms})$ in the left and at $274 \mathrm{~ms}( \pm 63 \mathrm{~ms})$ in the right hemisphere. At the time of the Pe activation (180-280 ms) the mean activation elicited by NoGo error trials $(17.1 \pm 13.4 \mathrm{nAm}$ in the left and $13.3 \pm 7.9 \mathrm{nAm}$ in the right hemisphere) was stronger than activation elicited during this time window by Go correct trials $(6.2 \pm 9.0 \mathrm{nAm}$ in the left and $3.0 \pm 3.1$ $\mathrm{nAm}$ in the right hemisphere, paired $t$-test between NoGo error and Go correct trials $p<0.002$ in the left and $p<0.001$ in the right hemisphere).

In summary, the response-locked signals in ERP channels revealed well-known negative ERN and positive Pe deflections associated with erroneous responses. During the ERN activation, 30-90 ms after the response onset, the right occipito-temporal border, right frontal area, left primary somatosensory area, and the anterior midline area were more strongly activated after NoGo error trials than Go correct trials. During the Pe deflection, 180-280 ms after response onset, bilateral temporal cortices and the left premotor area were more strongly activated to NoGo error trials than Go correct trials.

\section{Discussion}

We tracked electromagnetic signals while subjects were responding to complex visually presented frequent Go stimuli and withholding their response to infrequent non-target NoGo stimuli. We compared the stimulus-locked signals to Go correct trials and to successfully inhibited NoGo correct trials and response-locked signals to Go correct and NoGo error trials. The stimulus-locked signals in the ERP channels revealed an enhanced negative N2 and positive LPC deflections associated with NoGo correct trials. The response-locked signals revealed a negative ERN and positive Pe deflections associated with the NoGo error trials. The ERP findings are in line with several prior investigations (e.g., Nieuwenhuis et al., 2003; Amodio et al., 2008). Our most distinct MEG finding was the pronounced activation detected in the bilateral temporal cortices around the LPC and Pe 


\section{Minimum Current Estimates 30 - $90 \mathrm{~ms}$}

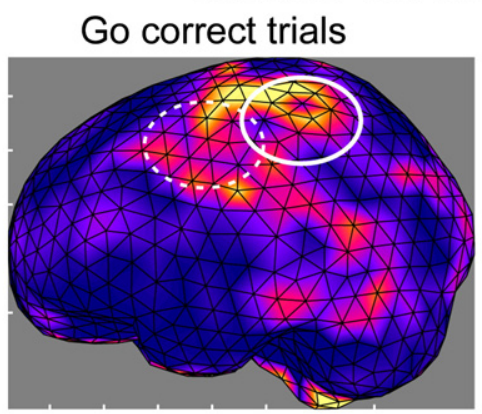

NoGo error trials
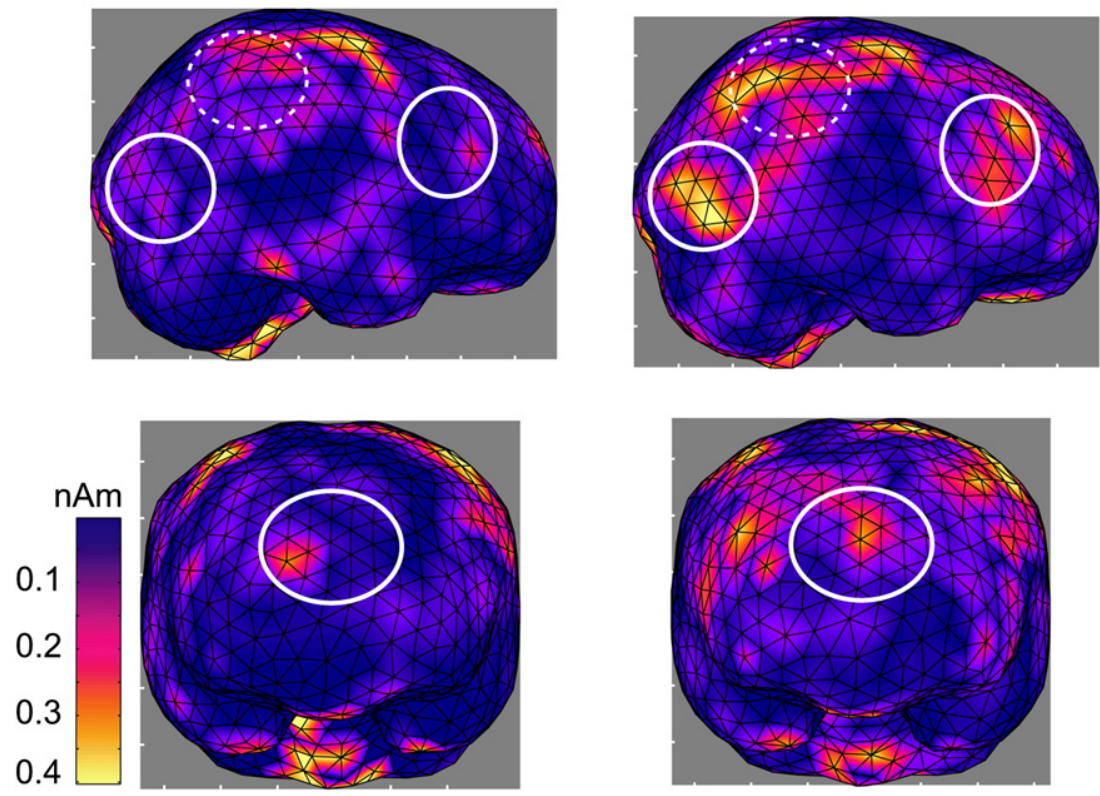

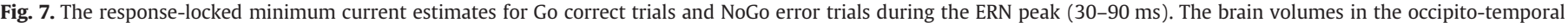

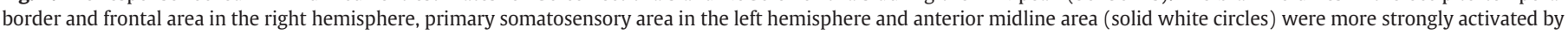

NoGo error than Go correct trails. The dashed circles indicate statistically non-significant differences between the two trials.

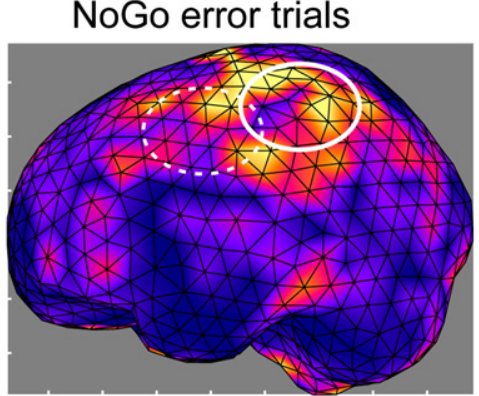

Minimum Current Estimates 180 - 280 ms

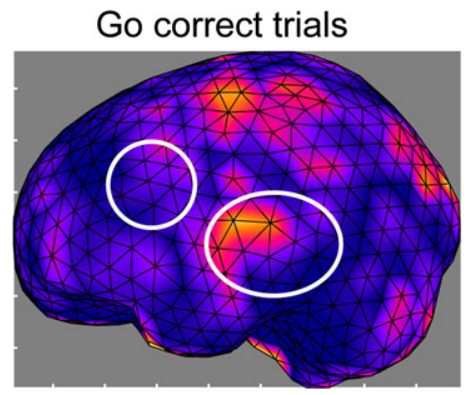

NoGo error trials
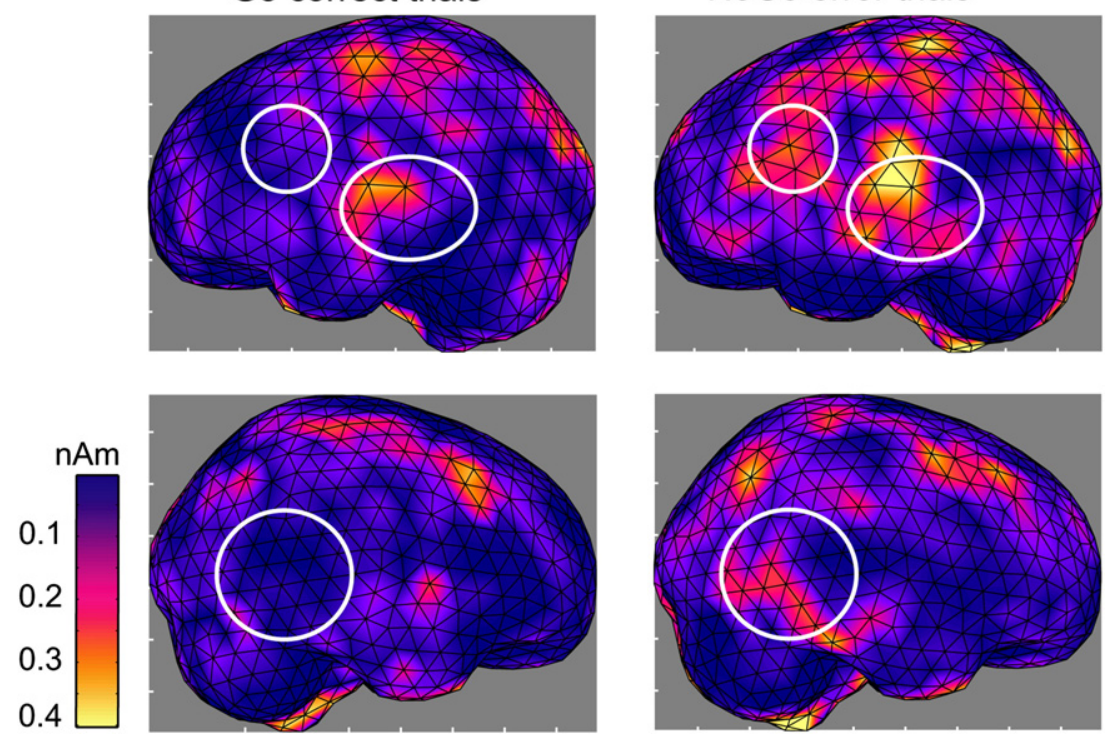

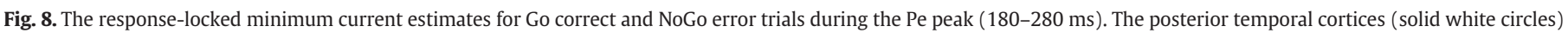
and the left frontal cortex showed stronger activation to NoGo error than Go correct trials. 

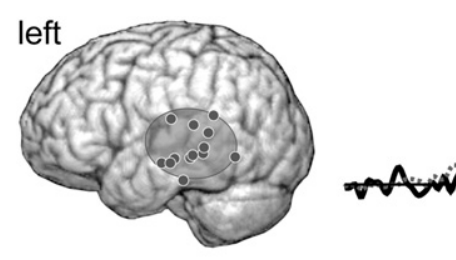

20
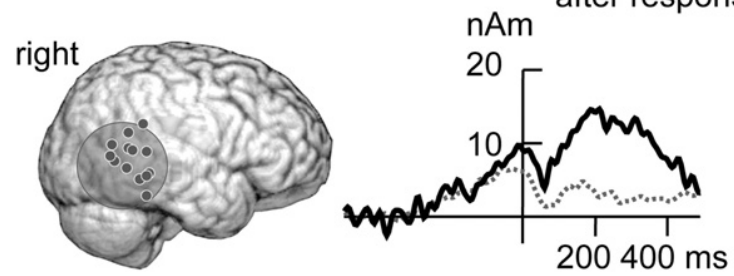

Fig. 9. Left : The distribution of equivalent current dipoles across all subjects used to explain the response-locked MEG data in the left and right temporal cortex. Right: The mean time courses of activation averaged across all subjects for Go correct and NoGo error trials in the left and right temporal areas.

deflections. Further, our MEG results suggest that during the N2 activation, right occipito-temporal border is more strongly activated to NoGo correct trials than to Go correct trials. During ERN, an enhanced signal after erroneous responses was detected in anterior midline area, in right frontal region and in the left primary somatosensory area, in addition to right posterior activation. The observed signal changes in anterior midline and somatosensory areas are consistent with previous MEG reports of error-related activation (Miltner et al., 2003; Stemmer et al., 2004). Significant transient increases in activity associated with errors have also been reported in dorsolateral prefrontal cortex and the left premotor cortex using fMRI (Carter et al., 1998).

The P3 component has been extensively studied for more than 40 years since its first description in the mid-60s by Sutton et al. (1965). Although many antecedent conditions, most notably the probability of occurrence, are well-known, the exact functional role and cortical basis of P3 has remained unclear. The positivity that follows errors, the Pe component, is either suggested to share the same neural source with P3 or, alternatively, relate specifically to error processing (Falkenstein, 2004) with possible neural source in the posterior cingulate regions (Vocat et al., 2008). The lack of consensus concerning the neural basis of the late positivities is also reflected in the varied interpretation of the $\mathrm{P} 3 /$ Pe findings in clinical populations (e.g., in ADHD, see Barry et al., 2003; Wiersema et al., 2009) and their connections to motivational or behavioral factors (Hajcak et al., 2004; Boksem et al., 2006).

Our results indicate that the bilateral posterior temporal cortex contributes to the late positive ERP components LPC and Pe. The temporal activity detected by MEG after infrequent visual stimuli was elicited irrespective of whether this stimulus was associated with correctly withheld response or followed by an erroneous response. Thus, purely error-related explanations of Pe (Falkenstein, 2004) are not feasible in explaining the bilateral temporal activation detected during both LPC and Pe in our study. Also, theories that view P3 as reflecting the decision on how to respond to the stimulus (Verleger et al., 2005) seem discordant with the current results showing that for the stimulus-locked NoGo error trials associated with a very rapid but incorrect response, the LPC response was very late (activation in the temporal areas peaked more than $200 \mathrm{~ms}$ after the response). Further, for the NoGo error trials the activation in the bilateral temporal areas was slightly delayed compared to the NoGo correct trials. This delay can be explained by the initial miscategorization of the stimulus (leading to an incorrect response), and as a consequence of this momentary misperception, the LPC was evoked only after the subject started to realize what the real category of the stimulus was. Although it may be possible to generate alternative accounts of this finding, our results are consistent with a view suggesting that the outcome of the internal decision-making processes, i.e., how the stimulus is classified by the subject, plays a key role in the P3 generation (Nieuwenhuis et al., 2005).

Patient studies have indicated that the temporo-parietal junction (inferior parietal lobule/superior temporal gyrus; Verleger et al., 1994) is critical for the elicitation of the P3 (more precisely the P3b component, see, e.g., Polich 2007, for novelty elicited P3a component). The right TPJ is also involved in the exploration of object-related information (Karnath et al., 2001) and is a part of the stimulus-driven attentional system (Corbetta and Shulman, 2002). When subjects are expecting certain stimuli the goal-directed dorsal attentional system is engaged. However, when unexpected stimulus is presented, the ventral stimulus-driven attentional system is used to redirect attention. In a meta-analysis of fMRI-studies, a ventral attention network, consistently more strongly activated by unexpected than expected targets, included areas in TPJ and superior temporal sulcus but only in the right hemisphere (He et al., 2007). Although our results lack the fine discriminatory power to detect the exact center of activation, the MEG activation detected in the superior temporal cortex during the P3/Pe peaks certainly overlaps with anatomical regions suggested to be involved in the stimulus-driven ventral attention network.

The normal functioning of the attentional networks can be disrupted due to brain damage. Unilateral spatial neglect is a common result of a lesion to the right side of the brain. Neglect patients seem to ignore visual stimuli in the left side of the visual space, although, when prompted, can direct their attention to the stimuli. Most common site of damage in these patients is in the right TPJ or right superior temporal gyrus (Vallar and Perani, 1987; Karnath et al., 2001). Thus, neglect is suggested to represent a dysfunction of the ventral TPJ system (Corbetta and Shulman, 2002, He et al., 2007). Our results, however, emphasize the bilateral nature of the visual activation system in normally functioning individuals despite the critical role of right hemisphere in neurological patients. This echoes the conclusions of studies showing bilaterally distributed MEG activation during language perception in neurologically intact individuals (Helenius et al., 1998; Helenius et al., 2002), despite the fact that left hemisphere damage often leads to language impairments.

The current MEG recordings were also used to investigate the neural basis of the negative ERN deflection evoked after erroneous responses and $\mathrm{N} 2$ deflection evoked after correct NoGo trials. In the current experiment we detected enhanced parallel activation after committed errors in the anterior midline area (possibly arising from ACC), in right frontal region and in posterior occipito-temporal area. This distributed pattern of activation could relate to the operation of the perception-action cycle that has been suggested to form the basis of adaptive behavior (Fuster, 2004). In this model, external and internal feedback is used to regulate the activation of the prefrontal areas and poly- and unimodal association areas of the posterior cortex connected with each other. Thus, as the internal response monitoring system in ACC is activated after a committed error, parallel activation is immediately generated in those cortical areas that are related to stimulus perception and response generation. These ERN-related results must, however, be evaluated cautiously. The analysis of errorrelated signals was challenged by the fairly low overall frequency of erroneous responses and the localization of the ERN was also exclusively based on distributed source analysis conducted on group-averaged data. Thus, further MEG studies are needed to judge the reliability and replicability of these findings.

During the N2 deflection, we did not detect similar parallel anteriorposterior activation as during the ERN response. Instead, activation evoked by NoGo trials that were associated with successfully inhibited responses was limited to the posterior occipito-temporal area. Thus, our results do not support the view that the ACC is activated during the N2 (Nieuwenhuis et al., 2003; Yeung et al., 2004; Amodio et al., 2008) but 
rather support the view that stimulus-driven factors are the basis of the N2 deflection (Nieuwenhuis et al., 2004; Folstein and Van Petten, 2008). It is impossible, however, to rule out that the absence of N2 activation in ACC reflects methodological limitations. Due to the relatively poor sensitivity of MEG to sources in deeper brain regions, the contribution of very weak sources could go unnoticed. Despite this reservation, our results provide compelling evidence that activation along the ventral visual stream, reflecting perceptual processes involved in the identification and discrimination of the complex visual stimuli (Goodale and Milner, 1992), contributes to signals detected in the N2 time window. This could also be the most straightforward explanation of why auditory NoGo N2 is strongly reduced compared to visual N2 (Falkenstein et al., 1995). Modality-specific N2 responses evoked by visual but not auditory stimuli have also been observed in the occipito-temporal cortex in intracranial recordings (Halgren et al., 1995b). Optimally, the contribution of different source areas to N2 and ERN and late positive components should be studied in the future by combining high-resolution EEG and MEG recordings.

\section{Conclusion}

In this study, we were able to gain valuable insight into cortical process of visual target detection and error commission by combining ERP and MEG recordings. MEG, owing to localization power and advanced analysis tools, was able to reveal neural correlates of negativities and late positivities associated with correct responses and errors. In particular our results demonstrate, that the bilateral posterior temporal cortices, part of the ventral attention network, contribute to late positive components. One of the earliest functional roles assigned to P3 components related this response to updating or revision of mental representation in working memory (Donchin, 1981; Donchin and Coles, 1988). If working memory is considered to provide workspace for manipulating and briefly maintaining contents of visual space in the spotlight of attention, then the working memory P3 hypothesis is in concordance with the stimulus-driven attentional system view of P3 activation. In the future, MEG studies are likely to deepen our knowledge on visual attentional and executive processes and their deficits in a similar manner that is has been recently used to elucidate the cortical basis of language-learning impairments (Helenius et al., 2009) and language development (Parviainen et al., 2006).

\section{Acknowledgments}

This work was supported by the Academy of Finland (National Centres of Excellence Programme 2006-2011, grant no 114794 to P.H., grants no 108410,217065 and 217998 to M.L., and grants no 109193 and 111799 to M.N.), Emil Aaltonen Foundation, and Otologic Research Foundation.

\section{Appendix A. Supplementary data}

Supplementary data associated with this article can be found, in the online version, at doi:10.1016/j.neuroimage.2010.06.059.

\section{References}

Amodio, D., Master, S.L., Yee, C.M., Taylor, S.E., 2008. Neurocognitive components of the behavioral inhibition and activation systems: Implications for theories of selfregulation. Psychophysiology 45, 11-19.

Barry, R.J., Johnstone, S.J., Clarke, A.R., 2003. A review of electrophysiology in attentiondeficit/hyperactivity disorder. II. Event-related potentials. Clin. Neurophysiol. 114 184-198.

Basile, L.F., Rogers, R.L., Simos, P.G., Papanicolaou, A.C., 1997. Magnetoencephalographic evidence for common sources of long latency fields to rare target and rare novel visual stimuli. Int. J. Psychophysiol. 25, 123-137.

Baudena, P., Halgren, E., Heit, G., Clark, J.M., 1995. Intracerebral potentials to rare target and distractor auditory and visual stimuli. III. Frontal cortex. Electroencephalogr. Clin. Neurophysiol. 94, 251-264.
Bledowski, C., Prvulovic, D., Hoechstetter, K., Scherg, M., Wibral, M., Goebel, R., Linden, D.E., 2004. Localizing P300 generators in visual target and distractor processing: a combined event-related potential and functional magnetic resonance imaging study. J. Neurosci. 24, 9353-9360.

Boksem, M.A., Tops, M., Wester, A.E., Meijman, T.F., Lorist, M.M., 2006. Error-related ERP components and individual differences in punishment and reward sensitivity. Brain Res. 1101, 92-101.

Botvinick, M.M., Cohen, J.D., Carter, C.S., 2004. Conflict monitoring and anterior cingulate cortex: an update. Trends Cogn. Sci. 8, 539-546.

Carter, C.S., Braver, T.S., Barch, D.M., Botvinick, M.M., Noll, D., Cohen, J.D., 1998. Anterior cingulate cortex, error detection, and the online monitoring of performance. Science 280, 747-749.

Corbetta, M., Shulman, G.L., 2002. Control of goal-directed and stimulus-driven attention in the brain. Nat. Rev. Neurosci. 3, 201-215.

Dehaene, S., Posner, M.I., Tucker, D.M., 1994. Localization of a neural system for error detection and compensation. Psychol. Sci. 5, 303-305.

Donchin, E., 1981. Presidential address, 1980. Surprise!...Surprise? Psychophysiology $18,493-513$.

Donchin, E., Coles, M.G.H., 1988. Is the P300 component a manifestation of cognitive updating? Behav. Brain Sci. 11, 357-427.

Downar, J., Crawley, A.P., Mikulis, D.J., Davis, K.D., 2000. A multimodal cortical network for the detection of changes in the sensory environment. Nat Neurosci. 3, 277-283.

Duncan-Johnson, C.C., Donchin, E., 1977. On quantifying surprise: the variation of eventrelated potentials with subjective probability. Psychophysiology 14, 456-467.

Eimer, M., 1993. Effects of attention and stimulus probability on ERPs in a go/nogo task. Biol. Psychol. 35, 123-138.

Falkenstein, M., 2004. ERP correlates of erroneous performance. In: Ullsperger, M., Falkenstein, M. (Eds.), Errors, conflicts and the brain. Current opinions on performance monitoring. Max-Planck-Institut fur Kognitions-und Neurowissenschaften, Leipzig, pp. 5-14.

Falkenstein, M., Hohnsbein, J., Hoormann, J., Blanke, L., 1991. Effects of crossmodeal divided attention on late ERP components. II. Error processing in choice reaction tasks. Electroencephalogr. Clin. Neurophysiol. 78, 447-455.

Falkenstein, M., Koshlykova, N.A., Kiroj, V.N., Hoormann, J., Hohnsbein, J., 1995. Late ERP components in visual and auditory Go/Nogo tasks. Electroencephalogr. Clin. Neurophysiol. 96, 36-43.

Folstein, J.R., Van Petten, C., 2008. Influence of cognitive control and mismatch on the N2 component of the ERP: a review. Psychophysiology 45, 152-170.

Fuster, J.M., 2004. Upper processing stages of the perception-action cycle. Trends Cogn. Sci. 8, 143-145.

Gehring, W.J., Coles, M.G.H., Meyer, D.E., Donchin, E., 1990. The error-related negativity: an event-related brain potential accompanying errors. Psychophysiology 27, 34 .

Gehring, W.J., Goss, B., Coles, M.G.H., Meyer, D.E., Donchin, E., 1993. A neural system for error detection and compensation. Psychol. Sci. 4, 385-390.

Goodale, M.A., Milner, A.D., 1992. Separate visual pathways for perception and action. Trends Neurosci. 15, 20-25.

Goto, Y., Brigell, M.G., Parmeggiani, L., 1996. Dipole-modeling of the visual evoked P300. J. Psychosom. Res. 41, 71-79.

Hajcak, G., McDonald, N., Simons, R.F., 2004. Error-related psychophysiology and negative affect. Brain Cogn. 56, 189-197.

Hajcak, G., Moser, J.S., Yeung, N., Simons, R.F., 2005. On the ERN and the significance of errors. Psychophysiology 42, 151-160.

Halgren, E., Baudena, P., Clarke, J.M., Heit, G., Liégeois, C., Chauvel, P., Musolino, A., 1995a. Intracerebral potentials to rare target and distractor auditory and visual stimuli. I. Superior temporal plane and parietal lobe. Electroencephalogr. Clin. Neurophysiol. 94, 191-220.

Halgren, E., Baudena, P., Clarke, J.M., Heit, G., Marinkovic, K., Devaux, B., Vignal, J.P., Biraben, A., 1995b. Intracerebral potentials to rare target and distractor auditory and visual stimuli. II. Medial, lateral and posterior temporal lobe. Electroencephalogr. Clin. Neurophysiol. 94, 229-250.

Halgren, E., Marinkovic, K., Chauvel, P., 1998. Generators of the late cognitive potentials in auditory and visual oddball tasks. Electroencephalogr. Clin. Neurophysiol. 106, 156-164.

Hämäläinen, M. Hari, R. Ilmoniemi, RJ. Knuutila, J., Lounasmaa, O.V., 1993. Magnetoencephalography-theory, instrumentation, and applications to noninvasive studies of the working human brain. Rev. Mod. Phys. 65, 413-497.

He, B.J., Snyder, A.Z., Vincent, J.L., Epstein, A., Shulman, G.L., Corbetta, M., 2007. Breakdown of functional connectivity in frontoparietal networks underlies behavioral deficits in spatial neglect. Neuron 53, 905-918.

Helenius, P., Parviainen, T., Paetau, R., Salmelin, R., 2009. Neural processing of spoken words in SLI and dyslexia. Brain 132, 1918-1927.

Helenius, P., Salmelin, R., Service, E., Connolly, J., Leinonen, S., Lyytinen, H., 2002. Cortical activation during spoken-word segmentation in non-reading-impaired and dyslexic adults. J. Neurosci. 22, 2936-2944.

Helenius, P., Salmelin, R., Service, E., Connolly, J.F., 1998. Distinct time courses of word and sentence comprehension in the left temporal cortex. Brain 121, 1133-1142.

Horiguchi, T., Ohta, K., Nishikawa, T., 2003. An MEG study of P300 activity during a color discrimination task 2: source localization study. Brain Dev. 25, 241-244.

Jodo, E., Kayama, Y., 1992. Relation of a negative ERP component to response inhibition in a Go/No-go task. Electroencephalogr. Clin. Neurophysiol. 82, 477-482.

Karnath, H.O., Ferber, S., Himmelbach, M., 2001. Spatial awareness is a function of the temporal not the posterior parietal lobe. Nature 411, 950-953.

Knight, R.T., Scabini, D., Woods, D.L., Clayworth, C.C., 1989. Contribution of the temporal-parietal junction to the auditory P3. Brain Res. 501, 109-116.

Kok, A., 1986. Effects of degradation of visual stimulation on components of the eventrelated potential (ERP) in Go/No-Go reaction tasks. Biol. Psychol. 23, 21-38. 
Marois, R., Leung, H., Gore, J.C., 2000. A stimulus-driven approach to object identity and location processing in the human brain. Neuron 25, 717-728.

McCarthy, G., Donchin, E., 1981. A metric for thought: a comparison of P300 latency and reaction time. Science $211,77-80$.

McCarthy, G., Luby, M., Gore, J., Goldman-Rakic, P., 1997. Infrequent events transiently activate human prefrontal and parietal cortex as measured by functional MRI. J. Neurophysiol. 77, 1630-1634.

Mecklinger, A., Maess, B., Opitz, B., Pfeifer, E., Cheyne, D., Weinberg, H., 1998. A MEC analysis of the P300 in visual discrimination tasks. Electroencephalogr. Clin. Neurophysiol. 108, 45-56.

Miltner, W.H.R., Lemke, U., Weiss, T., Holroyd, C., Scheffer, M.K., Coles, M.G.H., 2003. Implementation of error-processing in the human anterior cingulate cortex: a source analysis of the magnetic equivalent of the error-related negativity. Biol. Psychol. 64, 157-166.

Moores, K.A., Clark, C.R., Hadfield, J.L., Brown, G.C., Taylor, D.J., Fitzgibbon, S.P., Lewis, A.C. Weber, D.L., Greenblatt, R., 2003. Investigating the generators of the scalp recorded visuo-verbal P300 using cortically constrained source localization. Hum. Brain Mapp. $18,53-77$.

Nieuwenhuis, S., Aston-Jones, G., Cohen, J.D., 2005. Decision making, the P3, and the locus coeruleus norepinephrine system. Psychol. Bull. 131, 510-531.

Nieuwenhuis, S., Yeung, N., Cohen, J.D., 2004. Stimulus modality, perceptual overlap, and the Go/NoGo N2. Psychophysiology 41, 157-160.

Nieuwenhuis, S., Yeung, N., Van den Wildenberg, W., Ridderinkhof, K.R., 2003. Electrophysiological correlates of anterior cingulate function in a Go/NoGo task: effects of response conflict and trial-type frequency. Cogn. Affect. Behav. Neurosci. 3, $17-26$.

Overbeek, T.J.M., Nieuwenhuis, S., Ridderinkhof, K.R., 2005. Dissociable components of error processing: on the functional significance of the Pe vis-à-vis the ERN/Ne. J. Psychophysiol. 19, 319-329.

Parviainen, T., Helenius, P., Poskiparta, E., Niemi, P., Salmelin, R., 2006. Cortical sequence of word perception in beginning readers. J. Neurosci. 26, 6052-6061.

Pfefferbaum, A., Ford, J.M., Weller, B.J., Kopell, B.S., 1985. ERPs to response production and inhibition. Electroencephalogr. Clin. Neurophysiol. 60, 423-434.

Polich, J., 2007. Updating P300: an integrative theory of P3a and P3b. Clin. Neurophysiol. $118,2128-2148$.

Polich, J., Squire, L.R., 1993. P300 from amnesic patients with bilateral hippocampal lesions. Electroencephalogr. Clin. Neurophysiol. 86, 408-417.
Salmelin, R., Baillet, S., 2009. Electromagnetic brain imaging. Hum. Brain Mapp. 30 1753-1757.

Salmelin, R., Service, E., Kiesilä, P., Uutela, K., Salonen, O., 1996. Impaired visual word processing in dyslexia revealed with magnetoencephalography. Ann. Neurol. 40, 157-162.

Stemmer, B., Vihla, M., Salmelin, R., 2004. Activation of the human sensorimotor cortex during error-related processing: a magnetoencephalography study. Neurosci. Lett. 362, 44-47.

Stenbacka, L., Vanni, S., Uutela, K., Hari, R., 2002. Comparison of minimum current estimate and dipole modeling in the analysis of simulated activity in the human visual cortices. Neuroimage 16, 936-943.

Sutton, S., Braren, M., Zubin, J., John, E.R., 1965. Evoked-potential correlates of stimulus uncertainty. Science 150, 1187-1188.

Uutela, K., Hämäläinen, M., Somersalo, E., 1999. Visualization of magnetoencephalographic data using minimum current estimates. Neuroimage 10,173-180.

Vallar, G., Perani, D., 1987. The anatomy of spatial neglect in humans. In: Vallar, G. Perani, D., Jeannerod, M. (Eds.), Neurophysiological and neuropsychological aspects of spatial neglect. Elsevier, Amsterdam, pp. 235-258.

Vartiainen, J., Parviainen, T., Salmelin, R., 2009. Spatiotemporal convergence of semantic processing in reading and speech perception. J. Neurosci. 29, 9271-9280.

Verleger, R., Heide, W., Butt, C., Kömpf, D., 1994. Reduction of P3b in patients with temporo-parietal lesions. Cogn. Brain Res. 2, 103-116.

Verleger, R., Jaśkowski, P., Wascher, E., 2005. Evidence for an integrative role of P3b in linking reaction to perception. J. Psychophysiol. 19, 165-181.

Vocat, R., Pourtois, G., Vuilleumier, P., 2008. Unavoidable errors: a spatio-temporal analysis of time-course and neural sources of evoked potentials associated with error processing in a speeded task. Neuropsychology 46, 2545-2555.

Wiersema, J.R., van der Meere, J.J., Roeyers, H., 2009. ERP correlates of error monitoring in adult ADHD. J. Neural. Transm. 116, 371-379.

Wydell, T.N., Vuorinen, T., Helenius, P., Salmelin, R., 2003. Neural correlates of letterstring length and lexicality during reading in a regular orthography. J. Cogn. Neurosci. 15, 1052-1062.

Yamazaki, T., Kamijo, K., Kenmochi, A., Fukuzumi, S., Kiyuna, T., Takaki, Y., Kuroiwa, Y., 2000. Multiple equivalent current dipole source localization of visual event-related potentials during oddball paradigm with motor response. Brain Topogr. 12, 159-175.

Yeung, N., Cohen, J.D., Botvinick, M.M., 2004. The neural basis of error detection: conflict monitoring and the error-related negativity. Psychol. Rev. 111, 931-959. 